\title{
A GEOMETRICAL CHARACTERIZATION OF SINGLY GENERATED DOUGLAS ALGEBRAS
}

\author{
KEIJI IZUCHI
}

\begin{abstract}
If $B$ is a Douglas algebra with $B \supsetneqq H^{\infty}+C$, then $B$ is singly generated if and only if ball $\left(B / H^{\infty}+C\right)$ has an extreme point.
\end{abstract}

Let $H^{\infty}$ denote the space of boundary functions of bounded analytic functions in the open unit disk $D$. Let $L^{\infty}$ be the space of bounded measurable functions on $\partial D$ with respect to the normalized Lebesgue measure. A uniformly closed subalgebra between $H^{\infty}$ and $L^{\infty}$ is called a Douglas algebra. It is well known that $H^{\infty}+C$ is a Douglas algebra, where $C$ is the space of continuous functions on $\partial D$. By Chang and Marshall's theorem [2, 7], a Douglas algebra is generated by $H^{\infty}$ and complex conjugate of some inner functions. It is an interesting problem to give a characterization of a Douglas algebra which is generated by complex conjugate of a single inner function (see [7 and 3, p. 398]). Such a Douglas algebra will be called singly generated. Up to now, its characterization has not been known. We shall give a geometrical characterization of a singly generated Douglas algebra. For a subset $F$ of $L^{\infty}$, we denote by $[F]$ the uniformly closed subalgebra generated by $F$.

THEOREM. Let $B$ be a Douglas algebra with $H^{\infty}+C \varsubsetneqq B$. Then the following assertions are equivalent.

(i) There is an inner function I such that $B=\left[H^{\infty}, \bar{I}\right]$.

(ii) There is an extreme point of ball $\left(B / H^{\infty}+C\right)$.

For a Banach space $Y$, we denote by ball $(Y)$ the closed unit ball of $Y$. A point $x$ in ball $(Y)$ is called extreme if $\|x \pm y\| \leq 1$ and $y \in \operatorname{ball}(Y)$ imply $y=0$. Extreme points of ball $\left(B / H^{\infty}+C\right)$, where $B$ is a Douglas algebra, are studied in $[6,9]$.

For a Douglas algebra $B$, we write $M(B)$ as the maximal ideal space of $B$. We put $X=M\left(L^{\infty}\right)$. For a point $x$ in $M\left(H^{\infty}+C\right)$, we denote by $\mu_{x}$ the representing measure on $X$ for $x$, and by supp $\mu_{x}$ the support set for $\mu_{x}$. For an inner function $I$, we denote by $N(\bar{I})$ the weak $^{*}$-closure in $X$ of $\bigcup\left\{\operatorname{supp} \mu_{x}:\left.\left.\bar{I}\right|_{\operatorname{supp} \mu_{x}} \notin H^{\infty}\right|_{\operatorname{supp} \mu_{x}}\right\}$. We put $Q C=\left(H^{\infty}+C\right) \cap \overline{\left(H^{\infty}+C\right)}$. For some point $x$ in $X,\{y \in X ; f(y)=$ $f(x)$ for every $f \in Q C\}$ is called a $Q C$-level set.

To show our theorem, we need some lemmas.

LEMMA 1 [5, THEOREM 1]. If I is an inner function, then

(1) $Q \subset N(\bar{I})$ or $Q \cap N(\bar{I})=\varnothing$ for every $Q C$-level set $Q$, and

(2) $\left.\left.\bar{I}\right|_{Q} \notin H^{\infty}\right|_{Q}$ for every $Q C$-level set $Q$ with $Q \subset N(\bar{I})$.

LEMMA 2 [5, ProOF OF COROllaRY 4]. Let $I_{1}$ and $I_{2}$ be inner functions. Then $N\left(\bar{I}_{1}\right) \subset N\left(\bar{I}_{2}\right)$ if and only if $\left[H^{\infty}, \bar{I}_{1}\right] \subset\left[H^{\infty}, \bar{I}_{2}\right]$.

Received by the editors November 26, 1984 and, in revised form, June 24, 1985.

1980 Mathematics Subject Classification. Primary 30H05, 46B20, 46J30.

Key words and phrases. Douglas algebra, extreme point, inner function. 
Proof of THE THEOREM. The fact that (i) $\Rightarrow$ (ii) is already pointed out in [9]. By [9, Lemma 1], for an inner function $I$ there is an interpolating Blaschke product $b$ such that $\left[H^{\infty}, \bar{I}\right]=\left[H^{\infty}, \bar{b}\right]$, and $\bar{b}+H^{\infty}+C$ is an extreme point of ball $\left(\left[H^{\infty}, \bar{I}\right] / H^{\infty}+C\right)$ by $[6$, Theorem 5$]$.

To show the converse assertion, suppose that (i) is not true. Let $f \in B$ with $\left\|f+H^{\infty}+C\right\|=1$. Since $H^{\infty}+C$ has the best approximation property [1], we may assume $\|f\|=1$. We shall show that $f+H^{\infty}+C$ is not an extreme point of ball $\left(B / H^{\infty}+C\right)$. By Chang and Marshall's theorem, we have $\left[H^{\infty}, f\right]=$ $\left[H^{\infty}, \bar{I} ; I\right.$ is an inner function with $\left.\bar{I} \in\left[H^{\infty}, f\right]\right]$. Then there is an inner function $I_{0}$ such that $\left\|I_{0} f+H^{\infty}+C\right\|<1$ and $\bar{I}_{0} \in\left[H^{\infty}, f\right]$. we put $\alpha=1-\left\|I_{0} f+H^{\infty}+C\right\|$, then $\alpha>0$. We take a function $h$ with

$$
h \in H^{\infty}+C \text { and }\left\|I_{0} f+h\right\|=\left\|I_{o} f+H^{\infty}+C\right\| .
$$

Since $\left[H^{\infty}, \bar{I}_{0}\right] \subset B$, by our starting assumption there is an inner function $J$ such that

$$
\left[H^{\infty}, \bar{I}_{0}\right] \varsubsetneqq\left[H^{\infty}, \bar{J}\right] \subset B .
$$

By Lemma 2, we get $N\left(\bar{I}_{0}\right) \varsubsetneqq N(\bar{J})$. By Lemma 2(1), there is a $Q C$-level set $Q$ such that $Q \subset N(\bar{J})$ and $Q \cap N\left(\bar{I}_{0}\right)=\varnothing$. Then there is a function $q$ in $Q C$ satisfying

$$
0 \leq q \leq 1 \text { on } X, \quad q=1 \text { on } Q
$$

and

$$
q=0 \text { on a neighborhood of } N\left(\bar{I}_{0}\right) .
$$

To show our assertion, it is sufficient to prove that

$$
\bar{J} q \in B \text { and } \bar{J} q \notin H^{\infty}+C
$$

and

$$
\left\|f \pm \alpha \bar{J} q+H^{\infty}+C\right\| \leq 1 .
$$

(4) follows easily from (2) and Lemma 1(2). We shall prove that $\| f+\alpha \bar{J} q+H^{\infty}+$ $C \| \leq 1$; the other will be obtained by the same way. Let us take a measure $\mu$ on $X$ satisfying

$$
\|\mu\|=1 \text { and } \mu \perp H^{\infty}+C
$$

that is, $\mu$ is an annihilating measure for $H^{\infty}+C$ having the unit total variation, and

$$
\left\|f+\alpha \bar{J} q+H^{\infty}+C\right\|=\int_{X}(f+\alpha \bar{J} q) d \mu
$$

We put

$$
E=\{x \in X ; q(x)=0\} .
$$

Since $q \in Q C, E$ is a peak set for $H^{\infty}+C$. By the Glicksberg peak set theorem [3] and (6), we have $\left.\mu\right|_{E} \perp H^{\infty}+C$, so that

$$
\left.\mu\right|_{X \backslash E} \perp H^{\infty}+C .
$$


By (3), we can take $q_{0} \in Q C$ with $q_{0}=0$ on $N\left(\bar{I}_{0}\right)$ and $q_{0}=1$ on $X \backslash E$. Since $\left.\left.q_{0} \bar{I}_{0}\right|_{\text {supp } \mu_{x}} \in H^{\infty}\right|_{\text {supp } \mu_{x}}$ for every $x \in M\left(H^{\infty}+C\right)$, we have $q_{0} \bar{I}_{0} \in H^{\infty}+C$ by [8], so that $q_{0} \bar{I}_{0} h \in H^{\infty}+C$ by (1). By (9), we get

$$
\int_{X \backslash E} \bar{I}_{0} h d \mu=0 .
$$

Then

$$
\begin{aligned}
\left\|f+\alpha \bar{J} q+H^{\infty}+C\right\| & =\int_{X}(f+\alpha \bar{J} q) d \mu \quad \text { by }(7) \\
& =\int_{E}(f+\alpha \bar{J} q) d \mu+\int_{X \backslash E}(f+\alpha \bar{J} q) d \mu \\
& =\int_{E} f d \mu+\int_{X \backslash E}\left(f+\alpha \bar{J} q+\bar{I}_{0} h\right) d \mu \quad \text { by }(8) \text { and (10) } \\
& \leq\left\|\left.\mu\right|_{E}\right\|+\left\|\left.\mu\right|_{X \backslash E}\right\|\left\{\left\|f+\bar{I}_{0} h\right\|_{X \backslash E}+\alpha\right\} \quad \text { by }\|f\| \leq 1 \\
& \leq\left\|\left.\mu\right|_{E}\right\|+\left\|\left.\mu\right|_{X \backslash E}\right\|\left\{\left\|I_{0} f+h\right\|+\alpha\right\} \\
& \leq 1 \text { by (1) and (6). }
\end{aligned}
$$

Thus we get (5) and complete the proof.

REMARK. In the proof of (ii) $\Rightarrow$ (i), we actually prove that if $f \in B, \| I_{0} f+$ $H^{\infty}+C \|<1$ and $\left[H^{\infty}, \bar{I}_{0}\right] \varsubsetneqq B$ for some inner function $I_{0}$ with $\bar{I}_{0} \in\left[H^{\infty}, f\right]$, then $f+H^{\infty}+C$ is not an extreme point of ball $\left(B / H^{\infty}+C\right)$. Consequently, if $f+H^{\infty}+C$ is an extreme point of ball $\left(B / H^{\infty}+C\right)$, and if $I_{0}$ is an inner function such that $\bar{I}_{0} \in\left[H^{\infty}, f\right]$ and $\left\|I_{0} f+H^{\infty}+C\right\|<1$, then $B=\left[H^{\infty}, \bar{I}_{0}\right] \subset\left[H^{\infty}, f\right] \subset B$. Thus we get: If $f+H^{\infty}+C$ is an extreme point of ball $\left(B / H^{\infty}+C\right)$, then $B=\left[H^{\infty}, f\right]$.

\section{REFERENCES}

1. S. Axler, I. D. Berg, N. Jewell and A. L. Shields, Approximation by compact operators and the space $H^{\infty}+C$, Ann. of Math. (2) 109 (1979), 601-612.

2. S-Y. A. Chang, A characterization of Douglas subalgebras, Acta Math. 137 (1976), 81-89.

3. J. Garnett, Bounded analytic functions, Academic Press, New York, 1981.

4. I. Glicksberg, Measures orthogonal to algebras and sets of antisymmetry, Trans. Amer. Math. Soc. 105 (1962), 415-435.

5. K. Izuchi, $Q C$-level sets and quotients of Douglas algebras, J. Funct. Anal. 65 (1986).

6. K. Izuchi and Y. Izuchi, Extreme and exposed points in quotients of Douglas algebras by $H^{\infty}$ or $H^{\infty}+C$, Yokohama Math. J. 32 (1984), 45-54.

7. D. Marshall, Subalgebras of $L^{\infty}$ containing $H^{\infty}$, Acta Math. 137 (1976), 91-98.

8. D. Sarason, Functions of vanishing mean oscillation, Trans. Amer. Math. Soc. 207 (1975), 391-405.

9. R. Younis, Division in Douglas algebras and some applications, preprint.

DePARTMENT OF MATHEMATICS, KANAGAWA UNIVERSITY, YOKOHAMA 221, JAPAN 